
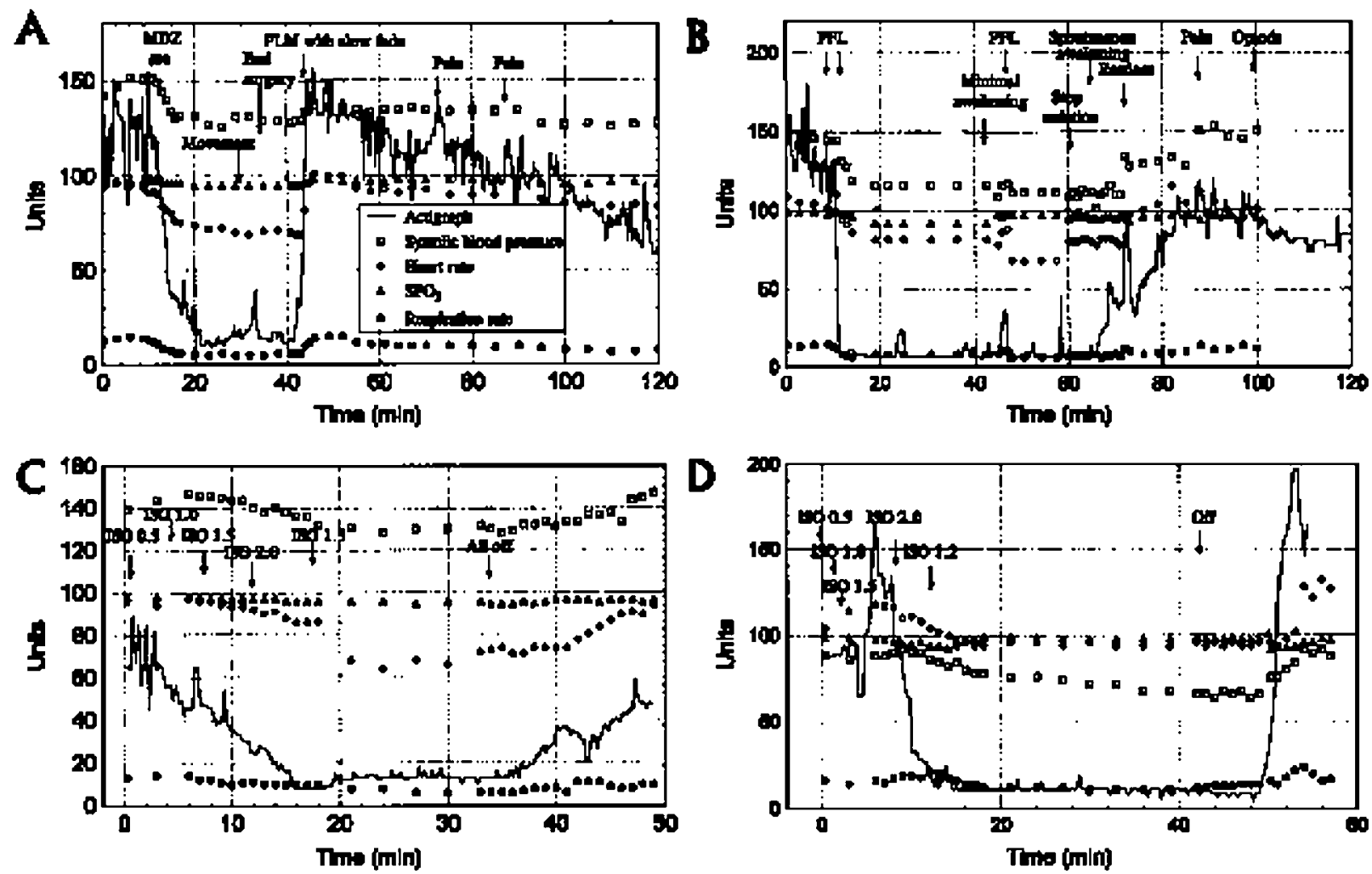

FIGURE 2 Actigrams and vital signs of patients undergoing surgery under monitored (epidural/spinal) sedation (A, B) and general anesthesia $(\mathrm{C}, \mathrm{D})$. Movement occurred during surgery in patient A. Note that the visible changes in the actigrams preceded equivalent changes in all other vital signs in all patients.

$\mathrm{MDZ}=$ midazolam; PFL=propofol; ISO=isoflurane; FLM=flumazenil; units: activity=arbitrary units; blood pressure=mmHg; heart rate=beats@min ${ }^{\mathrm{B}} ; \mathrm{SpO}_{2}=\%$; respiration rate=breaths@ $\min ^{\mathrm{B}}$.

2 Middlekoop HAM. Actigraphic Assessment of Sleep and Sleep Disorders. Delft: Eburon Publishers, 1994.

\section{Syringe contamination by propofol: a possible mechanism}

To the Editor:

Propofol, though a most popular induction agent providing an early awakening of anesthetized patients, ${ }^{1}$ has been shown to promote the growth of bacteria ${ }^{2}$ and fungi ${ }^{3}$ which might result in postoperative infection and sepsis. Breaking of ampoules, without taking aseptic measures, is the most common mode of extrinsic contamination. ${ }^{4}$ We report another possible source of such a contamination, which often goes unnoticed.

While administering propofol through an $i v$ cannula, a residual amount of medication stays in the drug port. The residual propofol, measured randomly in 50 patients, ranged between $0.05-0.15 \mathrm{~mL}$. When a subsequent drug is injected through the same port, the residual propofol migrates up into the syringe. Eventually, during prolonged procedures, the migrated propofol may lead to extrinsic contamination of subsequent drugs. This phenomenon is better noticed initially when propofol travels up in form of a thin streak followed by a "mushrooming effect" a la nuclear fission (Figure). Such contamination may contribute to postoperative infection and sepsis.

Manufacturers recommend that propofol be used within six hours of its handling, in a single patient, and not be stored thereafter. Sosis and Braverman ${ }^{3}$ demonstrated that the number of colony forming units of staphylococcus aureus were significantly greater when inoculated in sterile $0.9 \%$ saline when compared to solutions containing other anesthetics 


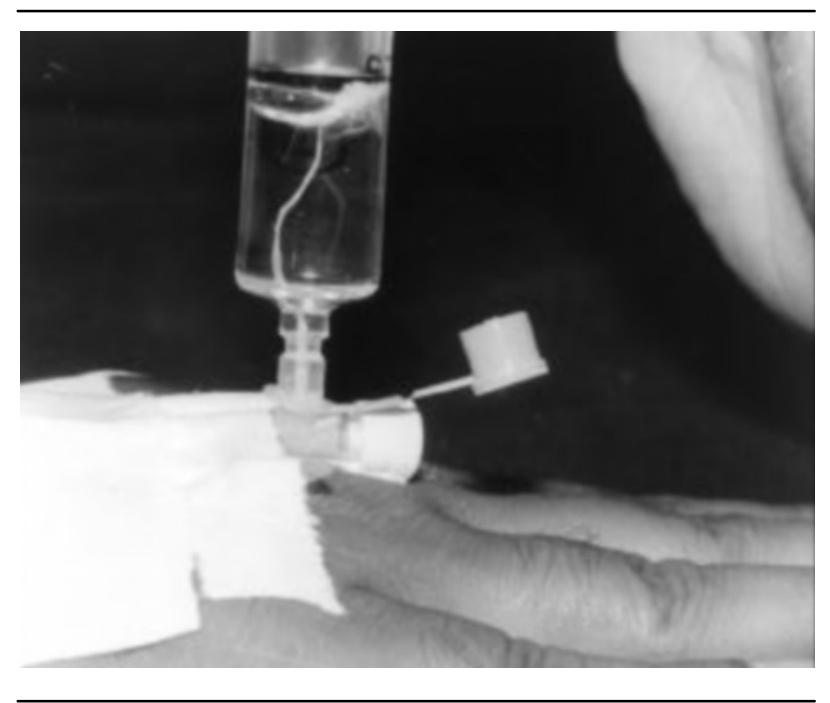

FIGURE Thin streak and "mushrooming effect" following migration of propofol into the subsequent drug syringe.

and summarized that saline does not possess any bacteriostatic property. Since water for injection and $0.9 \%$ saline are routine diluents for anesthetics, contamination by entrapped propofol is quite possible.

In conclusion, we endorse the recommendation to use a dedicated $i v$ cannula for administration of propofol not only to avoid its incompatibility with other anesthetic drugs,${ }^{5}$ but also to prevent its own contamination and that of the drugs in subsequent syringes.

Amitabh Dutta MD

S.K. Malhotra MD

Chandigarh, India

\section{References}

I Sebel PS, Lowdon JD. Propofol: a new intravenous anesthetic. Anesthesiology 1989; 71: 260-77.

2 Thomas DV. Propofol supports bacterial growth (Letter). Br J Anaesth 1991; 66: 274

3 Sosis MB, Braverman B. Growth of staphylococcus aureus in four intravenous anesthetics. Anesth Analg 1993; 77: 766-8.

4 Zacher AN, Zornow $M H$, Evans $G$ Drug contamination from opening glass ampoules. Anesthesiology 1991; $75: 893-5$.

5 Lamontagne C, Brouillette D, Hardy J-F. Incompatibility of propofol emulsion with anesthetic drugs (Letter). Anesthesiology 1998; 89: 1609.

\section{Opening an ampoule? Start from a scratch}

To the Editor:

Injuries to anesthesiologists ${ }^{1-3}$ during opening of drug ampoules still take place despite measures by the manufacturers to provide better snap off ampoules.

At our centre, we have been using a simple technique to break open the ampoules even when snap off ampoules were unheard of. As shown in the Figure, this technique consists of using the bottom edge of one ampoule to rub against the neck of the other ampoule - intended to be broken - till a grating sensation and sound is both felt and heard respectively and a small scratch mark appears at the rubbed site. Pressure over the top of the ampoule against the scratch mark neatly severs the top.

We continue to use a similar method even in propofol ampoules by rubbing the neck of the ampoule on the side opposite to the blue dot. By applying gentle pressure - as is instructed by the manufacturers - the ampoule can be easily snapped off, without a spicule, thereby decreasing the chance of injury.

Wasimul Huda MBBS

Rashid M. Khan MD

Aligarh, India

\section{References}

1 Ali PB. Persistent problem with propofol ampoules. Anaesthesia 1997; 52: 1020.

2 Morrison WJ. Another cracking idea. Anaesthesia 1999; 54: 307-8.

3 Palmer J, Ball D. Opening propofol ampoules. Anaesthesia 1998; 53: 416-7.

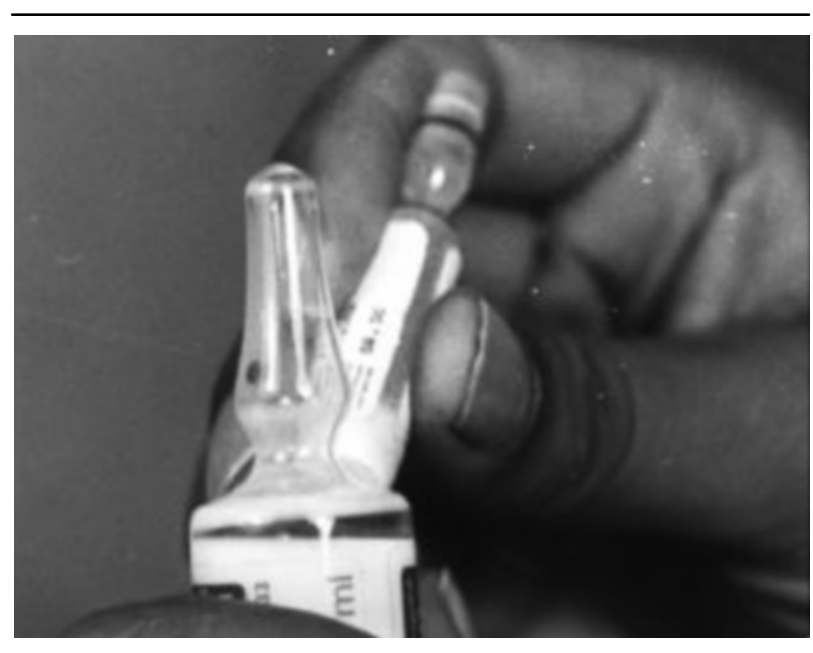

$\overline{\text { FIGURE }}$ 